\title{
Does higher screw density improve radiographic and clinical outcomes in adolescent idiopathic scoliosis? A systematic review and pooled analysis
}

\author{
*Ming Luo, MMed, ${ }^{1}$ Wengang Wang, MD, ${ }^{1}$ Mingkui Shen, MMed, ${ }^{1}$ Xin Luo, BS, ${ }^{2}$ and Lei Xia, MD1 \\ ${ }^{1}$ Institute of Spinal Deformity, The First Affiliated Hospital of Zhengzhou University, Zhengzhou, Henan; and 'Department of \\ Cardiovascular Medicine, The Central Hospital of Wuhan, Hubei, P. R. China
}

\begin{abstract}
OBJECTIVE The radiographic and clinical outcomes of low-density (LD) versus high-density (HD) screw constructs in patients with adolescent idiopathic scoliosis (AIS) treated with all-pedicle screw constructs are still controversial. A systematic review and pooled analysis were performed to compare radiographic, perioperative, and quality-of-life (QOL) outcomes and complications in patients with moderate AIS treated with LD or HD screw constructs.
\end{abstract}

METHODS The MEDLINE, Embase, and Web of Science databases were searched for English-language articles addressing LD versus HD screw constructs in AIS patients treated with all-pedicle screw constructs. The division of LD and HD groups was based on relative screw density and screw techniques. This systematic analysis strictly followed the PRISMA (Preferred Reporting Items for Systematic Reviews and Meta-Analyses) guidelines, and all articles included in the analysis met the criteria specified in the guidelines. Two reviewers independently assessed the quality of the studies using the Newcastle-Ottawa Scale. Date on radiographic, perioperative, and QOL outcomes and complications were extracted from the included studies.

RESULTS Twelve studies, involving a total of 827 patients (480 treated with LD constructs, 347 with HD), were analyzed-1 randomized controlled trial, 1 quasi-randomized controlled trial, and 10 retrospective studies. The patients' age at surgery, preoperative Cobb angle of the major curve, amount of thoracic kyphosis, and major curve flexibility were reasonably distributed, and no statistically significant differences were found. Regarding the outcomes at most recent follow-up, there were no significant differences in the Cobb angle of the major curve (mean difference $0.96{ }^{\circ}, 95 \% \mathrm{Cl}$ $-0.06^{\circ}$ to $1.98^{\circ}, p=0.06, l^{2}=1 \%$ ), major curve correction (mean difference $-0.72 \%, 95 \% \mathrm{Cl}-2.96 \%$ to $1.52 \%, p=0.53$, $I^{2}=0 \%$ ), thoracic kyphosis (mean difference $-1.67^{\circ}, 95 \% \mathrm{Cl}-4.59^{\circ}$ to $1.25^{\circ}, p=0.26, l^{2}=79 \%$ ), complications (odds ratio [OR] $0.66,95 \% \mathrm{Cl} 0.31-1.42, p=0.29, l^{2}=0 \%$ ), and QOL outcomes. Reduced operative time (mean difference -48.56 minutes, $95 \% \mathrm{Cl}-82.69$ to -14.43 minutes, $p=0.005, \mathrm{l}^{2}=87 \%$ ), blood loss (mean difference $-77.85 \mathrm{ml}, 95 \% \mathrm{Cl}$ -153.10 to $-2.60 \mathrm{ml}, \mathrm{p}=0.04, \mathrm{l}^{2}=0 \%$ ), and hospital charges (mean difference $-\$ 5.92 \mathrm{~K}, 95 \% \mathrm{Cl}-\$ 6.59 \mathrm{~K}$ to $-\$ 5.26 \mathrm{~K}, \mathrm{p}$ $<0.00001,1^{2}=0 \%$ ) were found in the LD group, compared with the HD group.

CONCLUSIONS LD and HD screw constructs are both associated with satisfactory radiographic and QOL outcomes with few complications. This study supports the use of LD screw constructs for the treatment of moderate AIS, because they resulted in reduced operative time, blood loss, and hospital charges while maintaining radiographic and QOL outcomes and complication rates similar to those achieved with HD screw constructs.

https://thejns.org/doi/abs/10.3171/2016.11.PEDS16334

KEY WORDS screw density; adolescent idiopathic scoliosis; low density; high density; spine; systematic review

$\mathrm{W}$ ITH better tridimensional corrections and mechanical fixation ${ }^{4,9,13,16,17}$ and lower revision rates than hybrid constructs, ${ }^{14,27}$ posterior pedicle screw constructs have become the primary surgical strategy for most cases of adolescent idiopathic scoliosis (AIS).
As surgical experience and techniques for placing pedicle screws have improved, pedicle screws have increasingly been used in the treatment of moderate AIS. A significant correlation between implant density (defined as number of screws per treated level) and major curve

ABBREVIATIONS AIS = adolescent idiopathic scoliosis; HD = high density; LD = low density; NOS = Newcastle-Ottawa Scale; $Q O L=$ qualify of life; SAQ = spinal appearance questionnaire; SRS = Scoliosis Research Society.

SUBMITTED June 6, 2016. ACCEPTED November 1, 2016.

INCLUDE WHEN CITING Published online February 3, 2017; DOI: 10.3171/2016.11.PEDS16334.

* Ming Luo and Wengang Wang contributed equally to this work. 
correction has been reported. In addition, high-density (HD) screw constructs have been shown to result in better restoration of thoracic kyphosis and better spinal appearance questionnaire (SAQ) scores. ${ }^{12,20,28}$ However, a large body of research has shown that low-density (LD) screw constructs can provide similar radiographic or clinical outcomes. 3,7,22-25,30,33 Furthermore, the number of pedicle screws placed has been shown to be an independent predictor of increased total cost for patients undergoing AIS correction procedures..$^{10}$ There could be significant benefits if the cost-effectiveness of LD screw constructs were to be proven, with comparable radiographic and clinical outcomes being achieved with fewer pedicle screws.

The purpose of this study was to compare the results of LD and HD screw constructs with respect to radiographic, perioperative, and quality-of-life (QOL) outcomes as well as complications in patients with AIS.

\section{Methods \\ Search Strategy}

This systematic review strictly followed the PRISMA (Preferred Reporting Items for Systematic Reviews and Meta-Analyses) 2009 guidelines, and articles were included only if they met the criteria specified in the guidelines. The MEDLINE, Embase, and Web of Science databases were searched for articles published during or before April 2016, using the following keywords: "implant density" OR "anchor density" OR "screw density" OR "low density" OR "limited density" OR "high density" with "adolescent idiopathic scoliosis." Two authors (M.L. and W.G.W.) independently screened the titles and abstracts of potentially eligible studies, and any differences were settled by mutual agreement. Related articles in databases and reference lists of the included articles were browsed to find additional studies that might qualify for inclusion.

\section{Inclusion and Exclusion Criteria}

Articles were included if they met all the following criteria: 1) inclusion of patients with an AIS diagnosis, 2) treatment with an all-pedicle screw construct, 3) controlled trial specifying LD and HD screw constructs, 4) published in the English-language literature, and 5) 1-year minimum follow-up. Articles were excluded if they 1) involved patients with congenital or neuromuscular scoliosis, 2) reported on the use of LD or HD screw constructs only; 3) involved an anterior-posterior approach.

Screw density was defined as the number of pedicle screws per vertebrae implanted. ${ }^{2}$ A screw density of 2.0 (the maximum) would mean that pedicle screws were placed bilaterally at each level of the construct (from the uppermost to the lowermost instrumented vertebra).

Instead of establishing a specific screw density threshold to distinguish the 2 groups, the definition of LD and HD screw constructs was relative. The primary principle of division was based on relative screw density. For example, in the study by Bharucha et al., ${ }^{2}$ the LD group was defined as patients with a screw density less than $1.3(<1.3$ screws per level) and the HD group was defined as those with a screw density greater than $1.3(>1.3$ screws per level). If the screw density was not recorded, the division was dependent on screw techniques. For example, in the study by Morr et al., ${ }^{23}$ a skipped pedicle screw pattern was allocated to the LD group, and a consecutive pedicle screw pattern was allocated to the HD group.

To avoid the confounding factors of hooks and wires, all-pedicle screw constructs were preferred in this systematic review. However, hooks were used in 2 potentially included studies. Bharucha et al. ${ }^{2}$ used thoracic pedicle screw constructs, which were defined as no more than 2 hooks anchored in the construct. In Kemppainen's study, a fusion construct consisted of pedicle screws except for 2 hooks at the uppermost instrumented vertebra. ${ }^{11}$ As the 2 studies reported, no more than 2 hooks were implanted in each patient, and we decided that the 2 studies could be included.

\section{Data Extraction}

Two reviewers (M.L. and M.K.S.) extracted data from the included papers. The extracted data included the following: 1) study ID, study design, publication data, study period, minimum follow-up, sample size, and age at surgery, 2) Cobb angle of the major curve and thoracic kyphosis (T5-T12) at preoperation and last follow-up, 3) Lenke classification, major curve flexibility, and major curve correction, 4) surgical techniques, screw density, operative time, blood loss, hospital charges, and implant costs, and 5) QOL outcomes and complications.

The operation data from the study by Bharucha et al. ${ }^{2}$ were not included in the analysis. Decreased operative time and blood loss were found in the HD group in his study, which obviously did not conform with common sense. Bharucha et al. explained that most of the cases in the LD group were their earlier cases, with the latter cases having an HD screw pattern, and increasing experience with implanting pedicle screws led to a decreased operative time. Finally, the data from the study by Liu et al. ${ }^{20}$ were divided into 2 subgroups according to the diameter of the titanium rods: low stiffness $(5.5 \mathrm{~mm})$ and high stiffness $(6.35 \mathrm{~mm})$.

\section{Quality Assessment}

The quality assessment of potentially included studies was independently appraised by 2 reviewers (M.L. and W.G.W.) using the Newcastle-Ottawa Scale (NOS), which was recommended in the Cochrane Handbook for Systematic Reviews of Interventions, version 5.1.0 (http:// handbook.cochrane.org), to evaluate the risk of bias of nonrandomized controlled studies. A study was judged on 3 broad criteria: the selection of the study groups, the comparability of the groups, and the ascertainment of the exposure for controlled studies. Studies with an NOS score lower than 6 were regarded as having a high risk of bias and were excluded.

\section{Statistical Analysis}

RevMan 5.3 (the Cochrane Collaboration) was used for pooling the data. Publication bias was assessed with funnel plots, and study heterogeneity was evaluated using Q statistics and $I^{2}$ (measure of heterogeneity, with lower values indicating less heterogeneity), and a fixed-effect model was used when $I^{2}$ was less than $50 \%$, whereas a 
random-effects model was used when $I^{2}$ was $50 \%$ or greater. Pooled mean differences and $95 \%$ confidence intervals (CIs) are presented for continuous variables, such as major curve correction. Dichotomous variables, such as complications, are presented as odds ratios (ORs) and 95\% CIs.

\section{Results}

\section{Description of the Study}

A total of 1583 potentially relevant studies were identified from electronic databases. Based on the inclusion and exclusion criteria and quality assessment, 12 studies with a total of 827 patients (480 LD, $347 \mathrm{HD}$ ) were included. One randomized controlled trial, 1 quasi-randomized controlled trial, and 10 retrospective studies were included., ${ }^{1,2}$, $8,11,12,19-21,23,29-31$ The flow diagram of study selection is shown in Fig. 1, and the characteristics and baseline parameters of the included studies are shown in Table 1. The age at surgery, Cobb angle of major curve, thoracic kyphosis, and preoperative major curve flexibility were reasonably distributed, and no significant differences were found.

Screw density was available in 9 studies, $1,2,8,11,12,20,21$, 30,31 and the primary division of the LD and HD groups was based on the relative screw density. The other 3 studies $^{19,23,29}$ only described screw patterns, with no specific screw density reported, and the comparison groups were based on screw placement techniques, such as consecutive versus interval/skipped pedicle screw fixation. The detailed information on screw density and screw placement techniques is shown in Table 2.

\section{Quality Assessment}

Using the NOS, 2 reviewers (M.L. and W.G.W.) rated the 12 controlled studies independently, and differences were resolved by consensus. The scores for all included studies were no less than 6 . The detailed quality-assessment scores are shown in Table 1. Publication bias was assessed with funnel plots, and the funnel plot of complications is symmetrical (Fig. 2).

\section{Radiographic Outcomes}

Radiographic outcomes for the Cobb angle of the major curve, major curve correction, and thoracic kyphosis at last follow-up were pooled and analyzed. Forest plots of radiographic outcomes are shown in Fig. 3.

Pooled data on the Cobb angle of the major curve were available in 7 studies, ${ }^{2,8,11,12,19,20,31}$ and no significant differences were found (mean difference $0.96^{\circ}, 95 \% \mathrm{CI}-0.06^{\circ}$ to $1.98^{\circ}, \mathrm{p}=0.06, I^{2}=1 \%$ ) between LD and HD groups. In addition, 3 other studies, ${ }^{23,29,30}$ involving a total of 298 patients, reported similar conclusions. Tsirikos and Subramanian ${ }^{30}$ reviewed 212 consecutive cases and observed that the LD and HD groups achieved comparable Cobb angles of the major curve (LD $19^{\circ}$ vs $\mathrm{HD} 20^{\circ}, \mathrm{p}=0.86$ ). Tao et al. ${ }^{29}\left(\mathrm{LD} 14^{\circ}\right.$ vs HD $\left.12^{\circ}, \mathrm{p}=0.19\right)$ and Morr et al..$^{23}$ (LD $17.7^{\circ}$ vs $\mathrm{HD} 17.3^{\circ}, \mathrm{p}=0.35$ ) also found no significant between-group differences in the Cobb angle of the major curve at the last follow-up.

Pooled data on major curve correction were extracted from 5 studies, ${ }^{2,8,11,20,21}$ and excellent coronal correction was achieved for both the LD and the HD groups (mean difference $-0.72 \%, 95 \% \mathrm{CI}-2.96 \%$ to $1.52 \%, \mathrm{p}=0.53, I^{2}=$ $0 \%$ ). Additionally, 3 other studies, ${ }^{1,23,30}$ involving a total of 306 patients, reported major curve correction. Auerbach et al. ${ }^{1}$ reviewed 54 Lenke Type 1 AIS cases and concluded

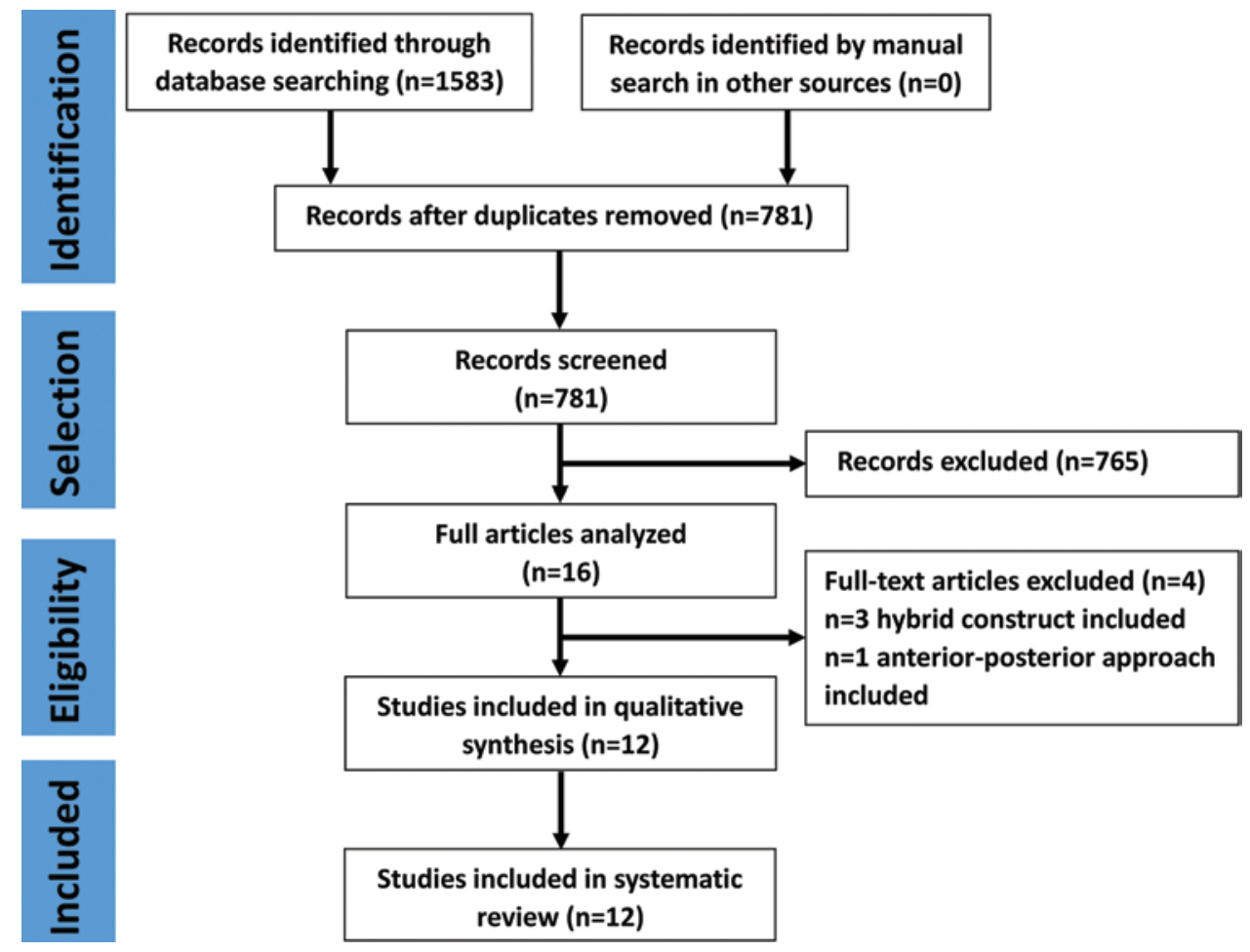

FIG. 1. Flow diagram of study selection. Figure is available in color online only. 
TABLE 1. Characteristics of the included studies

\begin{tabular}{|c|c|c|c|c|c|c|c|c|c|c|c|}
\hline $\begin{array}{l}\text { Authors } \\
\& \text { Year }\end{array}$ & $\begin{array}{l}\text { Study } \\
\text { Design }\end{array}$ & $\begin{array}{l}\text { Study } \\
\text { Period }\end{array}$ & $\begin{array}{l}\text { Min } \\
\text { FU } \\
\text { (yrs) }\end{array}$ & Group & $\begin{array}{c}\text { No. } \\
\text { of } \\
\text { Cases }\end{array}$ & Age $(y r s)^{*}$ & $\begin{array}{l}\text { Lenke Type } \\
(1 / 2 / 3 / 4 / 5 / 6)\end{array}$ & $\begin{array}{c}\text { Major } \\
\text { Curve }\left({ }^{\circ}\right)^{*}\end{array}$ & $\begin{array}{c}\text { Major Curve } \\
\text { Flexibility } \\
(\%)^{*}\end{array}$ & $\begin{array}{l}\text { Thoracic } \\
\text { Kyphosis } \\
\left({ }^{\circ}\right)^{*} \dagger\end{array}$ & $\begin{array}{l}\text { NOS } \\
\text { Score }\end{array}$ \\
\hline $\begin{array}{l}\text { Lonner et al., } \\
\qquad 2009\end{array}$ & Retro & 1995-2005 & 2 & $\begin{array}{l}\text { LD } \\
\text { HD }\end{array}$ & $\begin{array}{l}33 \\
34\end{array}$ & $\begin{array}{l}14.8 \pm 2.7 \\
13.8 \pm 1.7\end{array}$ & $\begin{array}{l}25 / 5 / 3 / 0 / 0 / 0 \\
23 / 2 / 4 / 0 / 0 / 5\end{array}$ & $\begin{array}{l}49 \pm 6 \\
54 \pm 9\end{array}$ & $\begin{array}{l}62 \pm 19 \\
53 \pm 22\end{array}$ & $\begin{array}{l}24 \pm 12 \\
26 \pm 11\end{array}$ & 8 \\
\hline $\begin{array}{l}\text { Auerbach et } \\
\text { al., } 2009\end{array}$ & Retro & 2001-2006 & 1.5 & $\begin{array}{l}\text { LD } \\
\text { HD }\end{array}$ & $\begin{array}{l}27 \\
27\end{array}$ & 15 & $\begin{array}{l}27 / 0 / 0 / 0 / 0 / 0 \\
27 / 0 / 0 / 0 / 0 / 0\end{array}$ & \multicolumn{3}{|c|}{$\begin{array}{l}\text { Similar baseline w/ respect to main thoracic } \\
\text { curve \& thoracic kyphosis }\end{array}$} & 6 \\
\hline Li et al., 2009 & $\begin{array}{l}\text { Quasi- } \\
\text { RCT }\end{array}$ & $2003-2005$ & 2 & $\begin{array}{l}\text { LD } \\
\text { HD }\end{array}$ & $\begin{array}{l}15 \\
15\end{array}$ & $\begin{array}{l}13.5 \pm 2.42 \\
13.2 \pm 2.40\end{array}$ & $\begin{array}{l}15 / 0 / 0 / 0 / 0 / 0 \\
15 / 0 / 0 / 0 / 0 / 0\end{array}$ & $\begin{array}{l}61.9 \pm 9.93 \\
60.7 \pm 11.7\end{array}$ & $\begin{array}{l}68.97 \pm 11.05 \\
69.17 \pm 10.45\end{array}$ & $\begin{array}{l}27.1 \pm 9.83 \\
27.3 \pm 10.6\end{array}$ & 8 \\
\hline $\begin{array}{c}\text { Tao et al., } \\
2010 \dagger\end{array}$ & Retro & 2000-2006 & 2 & $\begin{array}{l}\text { LD } \\
H D\end{array}$ & $\begin{array}{l}24 \\
22\end{array}$ & $\begin{array}{l}9.6(9.3-9.7) \ddagger \\
9.5(9.2-9.6) \ddagger\end{array}$ & $\begin{array}{l}\text { NR } \\
\text { NR }\end{array}$ & $\begin{array}{l}50(44-57) \ddagger \\
50(44-55) \ddagger\end{array}$ & $\begin{array}{l}\text { NR } \\
\text { NR }\end{array}$ & $\begin{array}{l}\text { NR } \\
\text { NR }\end{array}$ & 8 \\
\hline $\begin{array}{l}\text { Tsirikos \& } \\
\text { Subrama- } \\
\text { nian, } 2012\end{array}$ & Retro & $2006-2010$ & 2.5 & $\begin{array}{l}\text { LD } \\
\text { HD }\end{array}$ & $\begin{array}{r}161 \\
51\end{array}$ & $\begin{array}{l}14.8 \\
14.8\end{array}$ & $\begin{array}{l}44 / 12 / 49 / 9 / 32 / 15 \\
15 / 4 / 18 / 5 / 3 / 6\end{array}$ & $\begin{array}{l}65 \\
68\end{array}$ & $\begin{array}{l}39 \\
43\end{array}$ & $\begin{array}{l}30 \\
26\end{array}$ & 8 \\
\hline $\begin{array}{c}\text { Gotfryd \& } \\
\quad \text { Avanzi, } \\
2013\end{array}$ & $\mathrm{RCT}$ & 2009-2011 & 2 & $\begin{array}{l}\text { LD } \\
\text { HD }\end{array}$ & $\begin{array}{l}23 \\
23\end{array}$ & $\begin{array}{l}11-18 \S \\
11-18 \S\end{array}$ & $\begin{array}{l}23 / 0 / 0 / 0 / 0 / 0 \\
23 / 0 / 0 / 0 / 0 / 0\end{array}$ & $\begin{array}{l}57.2 \pm 10.6 \\
59.7 \pm 6.6\end{array}$ & $\begin{array}{l}53.5 \pm 17.8 \\
44.7 \pm 12.2\end{array}$ & $\begin{array}{c}19.1 \pm 8.1 \\
23.5 \pm 11.8\end{array}$ & 8 \\
\hline $\begin{array}{c}\text { Bharucha et } \\
\text { al., } 2013\end{array}$ & Retro & 2001-2007 & 2 & $\begin{array}{l}\text { LD } \\
\text { HD }\end{array}$ & $\begin{array}{l}57 \\
34\end{array}$ & $\begin{array}{l}15.4 \pm 2.4 \\
15.4 \pm 2.3\end{array}$ & $\begin{array}{l}57 / 0 / 0 / 0 / 0 / 0 \\
34 / 0 / 0 / 0 / 0 / 0\end{array}$ & $\begin{array}{l}48 \pm 6 \\
48 \pm 6\end{array}$ & $\begin{array}{l}54 \pm 17 \\
48 \pm 18\end{array}$ & $\begin{array}{l}24 \pm 13 \\
24 \pm 12\end{array}$ & 8 \\
\hline $\begin{array}{l}\text { Liu et al., } \\
\qquad 2015 \text { (L-F) }\end{array}$ & & & 1 & $\begin{array}{l}\text { LD } \\
\text { HD }\end{array}$ & $\begin{array}{l}18 \\
17\end{array}$ & & $\begin{array}{l}18 / 0 / 0 / 0 / 0 / 0 \\
17 / 0 / 0 / 0 / 0 / 0\end{array}$ & $\begin{array}{l}51.03 \pm 16.51 \\
52.79 \pm 17.69\end{array}$ & $\begin{array}{l}40.03 \pm 20.13 \\
46.91 \pm 16.67\end{array}$ & $\begin{array}{l}22.36 \pm 8.05 \\
20.84 \pm 4.79\end{array}$ & \\
\hline $\begin{array}{l}\text { Liu et al., } \\
\qquad 2015(\mathrm{H}-\mathrm{F})\end{array}$ & Retro & & & $\begin{array}{l}\text { LD } \\
\text { HD }\end{array}$ & $\begin{array}{l}22 \\
20\end{array}$ & & $\begin{array}{l}22 / 0 / 0 / 0 / 0 / 0 \\
20 / 0 / 0 / 0 / 0 / 0\end{array}$ & $\begin{array}{l}48.86 \pm 7.90 \\
44.80 \pm 9.75\end{array}$ & $\begin{array}{l}44.82 \pm 20.18 \\
45.66 \pm 24.93\end{array}$ & $\begin{array}{r}22.35 \pm 9.06 \\
21.41 \pm 7.99\end{array}$ & 8 \\
\hline $\begin{array}{l}\text { Morr et al., } \\
2015\end{array}$ & Retro & NR & 2 & $\begin{array}{l}\text { LD } \\
H D\end{array}$ & $\begin{array}{l}20 \\
20\end{array}$ & $\begin{array}{l}14.2 \\
13.8\end{array}$ & $\begin{array}{l}20 / 0 / 0 / 0 / 0 / 0 \\
20 / 0 / 0 / 0 / 0 / 0\end{array}$ & $\begin{array}{l}51.7 \\
52.1\end{array}$ & $\begin{array}{l}\mathrm{NR} \\
\mathrm{NR}\end{array}$ & $\begin{array}{l}23 \\
19.9\end{array}$ & 8 \\
\hline $\begin{array}{l}\text { Wang et al., } \\
2016\end{array}$ & Retro & 2011-2014 & 1 & $\begin{array}{l}\text { LD } \\
\text { HD }\end{array}$ & $\begin{array}{l}16 \\
20\end{array}$ & $\begin{array}{l}14.4 \pm 1.6 \\
15.4 \pm 1.6\end{array}$ & $\begin{array}{l}20 / 0 / 0 / 0 / 0 / 0 \\
16 / 0 / 0 / 0 / 0 / 0\end{array}$ & $\begin{array}{l}44.6 \pm 4.6 \\
46.9 \pm 5.5\end{array}$ & $\begin{array}{l}62.4 \pm 2.1 \\
63.6 \pm 4.1\end{array}$ & $\begin{array}{l}21.5 \pm 6.5 \\
22.9 \pm 6.9\end{array}$ & 8 \\
\hline $\begin{array}{l}\text { Ketenci et al., } \\
2016\end{array}$ & Retro & $2008-2013$ & 1 & $\begin{array}{l}\text { LD } \\
\text { HD }\end{array}$ & $\begin{array}{l}38 \\
38\end{array}$ & $\begin{array}{c}15.1 \pm 2.1 \\
15.4 \pm 2.3\end{array}$ & $\begin{array}{l}38 / 0 / 0 / 0 / 0 / 0 \\
38 / 0 / 0 / 0 / 0 / 0\end{array}$ & $\begin{array}{l}52.8 \pm 10.7 \\
57.2 \pm 13.5\end{array}$ & $\begin{array}{l}38.7 \pm 8.9 \\
42.3 \pm 10.2\end{array}$ & $\begin{array}{l}28.9 \pm 11.7 \\
30.2 \pm 10.3\end{array}$ & 8 \\
\hline $\begin{array}{l}\text { Kemppainen } \\
\text { et al., } 2016\end{array}$ & Retro & 2007-2011 & 2 & $\begin{array}{l}\text { LD } \\
\text { HD }\end{array}$ & $\begin{array}{l}26 \\
26\end{array}$ & $\begin{array}{l}14.7 \pm 1.5 \\
14.8 \pm 1.9\end{array}$ & $\begin{array}{l}17 / 6 / 2 / 0 / 0 / 1 \\
17 / 3 / 0 / 1 / 0 / 5\end{array}$ & $\begin{array}{l}54.3 \pm 5.8 \\
59.5 \pm 8.2\end{array}$ & $\begin{array}{l}58 \pm 15 \\
57 \pm 15\end{array}$ & $\begin{array}{l}23.4 \pm 15.3 \\
21.8 \pm 13.3\end{array}$ & 7 \\
\hline
\end{tabular}

$\mathrm{H}-\mathrm{F}=$ high-stiffness rod group; L-F = low-stiffness rod group; NR = not reported; $\mathrm{RCT}$ = randomized controlled trial; retro = retrospective.

* Expressed as mean alone or mean \pm SD unless otherwise indicated.

† T5-12.

‡ Median (interquartile range).

$\S$ Full range.

that there were no significant differences between LD and HD cases with respect to correction of the Cobb angle of the major curve (LD 64\% vs HD 66\%, p =0.56), which was similar to the findings of Tsirikos and Subramanian ${ }^{30}$ (LD $70 \%$ vs HD 71\%, $\mathrm{p}=0.85$ ) and Morr et al. ${ }^{23}$ (LD $66.6 \%$ vs HD $66.9 \%, \mathrm{p}=0.92$ ).

Pooled data on thoracic kyphosis were available in 8 studies, involving a total of 475 patients. , $^{2,11,12,19-21,31}$ There were no significant differences in thoracic kyphosis (mean difference $-1.67^{\circ}, 95 \% \mathrm{CI}-4.59^{\circ}$ to $1.25^{\circ}, \mathrm{p}=0.26, I^{2}$ = 79) between the 2 groups. Furthermore, the results of Tsirikos and Subramanian ${ }^{30}\left(\right.$ LD $27^{\circ}$ vs HD 20, $\mathrm{p}=0.15$ ) and Morr et al..$^{23}$ (LD $19.4^{\circ}$ vs HD $16.9^{\circ}, \mathrm{p}=0.37$ ) were consistent with the pooled values.

\section{Perioperative Outcomes}

Operative time, blood loss, and hospital charges were evaluated in the pooled analysis. Forest plots of these perioperative outcomes are shown in Fig. 4.
Pooled data on operative time were extracted from 4 studies. ${ }^{11,19,21,31}$ Reduced operative time (mean difference -48.56 minutes, $95 \%$ CI -82.69 to -14.43 minutes, $\mathrm{p}=$ $0.005, I^{2}=87 \%$ ) was found in the LD group. In addition, Tsirikos and Subramanian ${ }^{30}$ reviewed 212 consecutive cases and reached the same conclusion (LD 240 minutes vs HD 320 minutes, p < 0.001). However, Gotfryd and Avanzi ${ }^{8}$ reported that there was no significant difference in operative time between the 2 groups (LD 244.8 minutes vs HD 275.9 minutes, $\mathrm{p}=0.179$ ).

Pooled data on blood loss were available in 4 studies. ${ }^{11,19,21,31}$ Reduced blood loss (mean difference $-77.85 \mathrm{ml}$, $95 \% \mathrm{CI}-153.10$ to $-2.60 \mathrm{ml}, \mathrm{p}=0.04, I^{2}=0 \%$ ) was also observed in the LD group. Moreover, the results of Tsirikos and Subramanian ${ }^{30}$ were consistent with the pooled data (LD $1343 \mathrm{ml}$ vs HD $1679 \mathrm{ml}, \mathrm{p}$ = 0.04). However, Gotfryd and Avanzi found that blood loss was comparable in the 2 groups (LD $655.7 \mathrm{ml}$ vs HD $687.0 \mathrm{ml}, \mathrm{p}=0.608) .^{8}$

Pooled data on hospital charges were available from 2 
TABLE 2. Surgical techniques and perioperative outcomes of the included studies

\begin{tabular}{|c|c|c|c|c|}
\hline Authors \& Year & Group & Screw Techniques & $\begin{array}{c}\text { Screw } \\
\text { Density* }\end{array}$ & $\begin{array}{l}\text { Mean Implant } \\
\text { Cost (\$) }\end{array}$ \\
\hline \multirow[t]{2}{*}{ Lonner et al., 2009} & LD & Polyaxial PS + cross-links & $1.06 \pm 0.11$ & NR \\
\hline & $\mathrm{HD}$ & Monoaxial PS + cross-links & $1.69 \pm 0.17$ & NR \\
\hline \multirow{2}{*}{$\begin{array}{l}\text { Auerbach et al., } \\
2009\end{array}$} & LD & Thoracic PS constructs (screw density < 1.1) + cross-links & 1.0 & 10,300 \\
\hline & $\mathrm{HD}$ & Thoracic PS constructs (screw density $>1.1)+$ cross-links & 1.3 & 12,027 \\
\hline \multirow[t]{2}{*}{ Li et al., 2009} & LD & Bilat interval PS fixation + cross-links & NR & NR \\
\hline & $\mathrm{HD}$ & Consec PS fixation on concave side \& interval on convex side + cross-links & NR & NR \\
\hline \multirow[t]{2}{*}{ Tao et al., 2010} & LD & Bilat interval PS fixation + cross-links & NR & NR \\
\hline & $\mathrm{HD}$ & Consec PS fixation on concave side \& interval on convex side + cross-links & NR & NR \\
\hline \multirow{2}{*}{$\begin{array}{l}\text { Tsirikos \& Subra- } \\
\text { manian, } 2012\end{array}$} & LD & Unilat segmental PS technique & 1.38 & Reduced $35 \%$ in \\
\hline & $\mathrm{HD}$ & Bilat segmental PS technique & 2 & LD group \\
\hline \multirow{2}{*}{$\begin{array}{l}\text { Gotfryd \& Avanzi, } \\
2013\end{array}$} & LD & 4 PS in base, 3 in central vertebrae, \& 3 in superior vertebrae + cross-links & 1.20 & 6,687 \\
\hline & $\mathrm{HD}$ & Consec PS fixation on concave side \& interval on convex side + cross-links & 1.61 & 8,195 \\
\hline \multirow{2}{*}{$\begin{array}{l}\text { Bharucha et al., } \\
2013\end{array}$} & LD & Straight-ahead PS technique (screw density $<1.3)+$ hooks $(\leq 2)+$ cross-links & $1.1 \pm 0.1$ & 10,819 \\
\hline & $\mathrm{HD}$ & Straight-ahead PS technique (screw density $>1.3)+$ hooks $(\leq 2)+$ cross-links & $1.6 \pm 0.2$ & 13,272 \\
\hline \multirow{2}{*}{$\begin{array}{l}\text { Liu et al., } 2015 \\
\quad(L-F)\end{array}$} & LD & 5.5-mm titanium rods + PS fixation (screw density $<1.2)$ & $1.08 \pm 0.06$ & NR \\
\hline & $\mathrm{HD}$ & 5.5-mm titanium rods + PS fixation (screw density $\geq 1.2$ ) & $1.36 \pm 0.14$ & NR \\
\hline \multirow{2}{*}{$\begin{array}{l}\text { Liu et al., } 2015 \\
\qquad(\mathrm{H}-\mathrm{F})\end{array}$} & LD & 6.35-mm titanium rods + PS fixation (screw density < 1.2) & $1.02 \pm 0.10$ & NR \\
\hline & $\mathrm{HD}$ & 6.35-mm titanium rods + PS fixation (screw density $\geq 1.2$ ) & $1.32 \pm 0.12$ & NR \\
\hline \multirow[t]{2}{*}{ Morr et al., 2015} & LD & Consec PS fixation on concave side \& interval on convex side & NR & 13,300 \\
\hline & $\mathrm{HD}$ & Bilat consec PS fixation & NR & 19,500 \\
\hline \multirow[t]{2}{*}{ Wang et al., 2016} & LD & Bilat interval PS fixation & 1.21 & NR \\
\hline & $\mathrm{HD}$ & Consec PS fixation on concave side \& interval on convex side & 1.60 & NR \\
\hline \multirow[t]{2}{*}{ Ketenci et al., 2016} & LD & Consec PS fixation on concave side \& interval on convex side + cross-links & $1.14 \pm 0.24$ & NR \\
\hline & $\mathrm{HD}$ & Bilat consec PS fixation + cross-links & 2.0 & NR \\
\hline \multirow{2}{*}{$\begin{array}{l}\text { Kemppainen et al., } \\
2016\end{array}$} & LD & Bilat interval PS fixation + uppermost hooks & $1.1 \pm 0.06$ & NR \\
\hline & $\mathrm{HD}$ & Consec PS fixation on concave side \& interval on convex side + uppermost hooks & $1.5 \pm 0.22$ & NR \\
\hline
\end{tabular}

Consec $=$ consecutive; $P S=$ pedicle screw.

* Mean number of screws per level $( \pm S D)$.

studies. ${ }^{11,31}$ Decreased hospital charges (mean difference $-\$ 5.92$ thousand, $95 \% \mathrm{CI}-\$ 6.59$ thousand to $-\$ 5.26$ thousand, $\mathrm{p}<0.00001, I^{2}=0 \%$ ) were found in the LD group, representing a cost savings of approximately $\$ 6000$ per patient. Furthermore, 5 other studies, involving 443 patients, reported implant costs. ${ }^{1,2,8,23,30}$ All 5 studies demonstrated that implant costs were significantly higher in the HD group; detailed information is shown in Table 2.

\section{QOL Outcomes}

The reviewed studies used a variety of methodologies to report QOL outcomes, including the SRS (Scoliosis Research Society)-22 questionnaire, the SRS-30 questionnaire, and the SAQ, which restricted the pooling analysis. In total, 6 studies, involving a total of 419 patients, recorded QOL outcomes at the last follow-up., ${ }^{1,2,8,12,23,30}$ Although Ketenci et al. ${ }^{12}$ reported a significantly better score in the SAQ appearance domain $(\mathrm{p}=0.035)$ for the HD group, no significant differences were found in any SRS-22 domain scores in his study. Furthermore, the SRS-22 questionnaire was adopted from 4 other studies, and all of them reported homogeneous outcomes. ${ }^{1,2,23,30}$ SRS-30 scores were reported by Gotfryd and Avanzi, ${ }^{8}$ and similar scores were found in the LD and HD groups for all 5 domains between. Detailed information on QOL outcomes is shown in Table 3.

\section{Complications}

Pooled data on complications were available from 7 studies, $1,2,8,11,19,30,31$ and the forest plot is shown in Fig. 5. No significant differences were observed between the LD and HD groups (OR 0.66, 95\% CI 0.31-1.42, $\mathrm{p}=0.29, I^{2}$ $=0 \%$ ). Additionally, no screw-related complications were

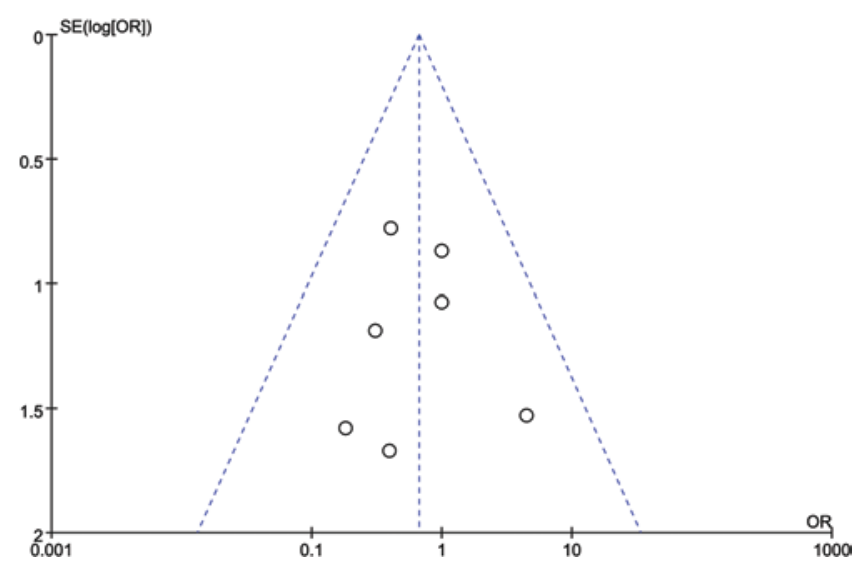

FIG. 2. Funnel plot of complications. The $x$-axis indicates odds ratio (OR) of complications, and the $y$-axis indicates SE(log[OR]). SE = standard error. Figure is available in color online only. 


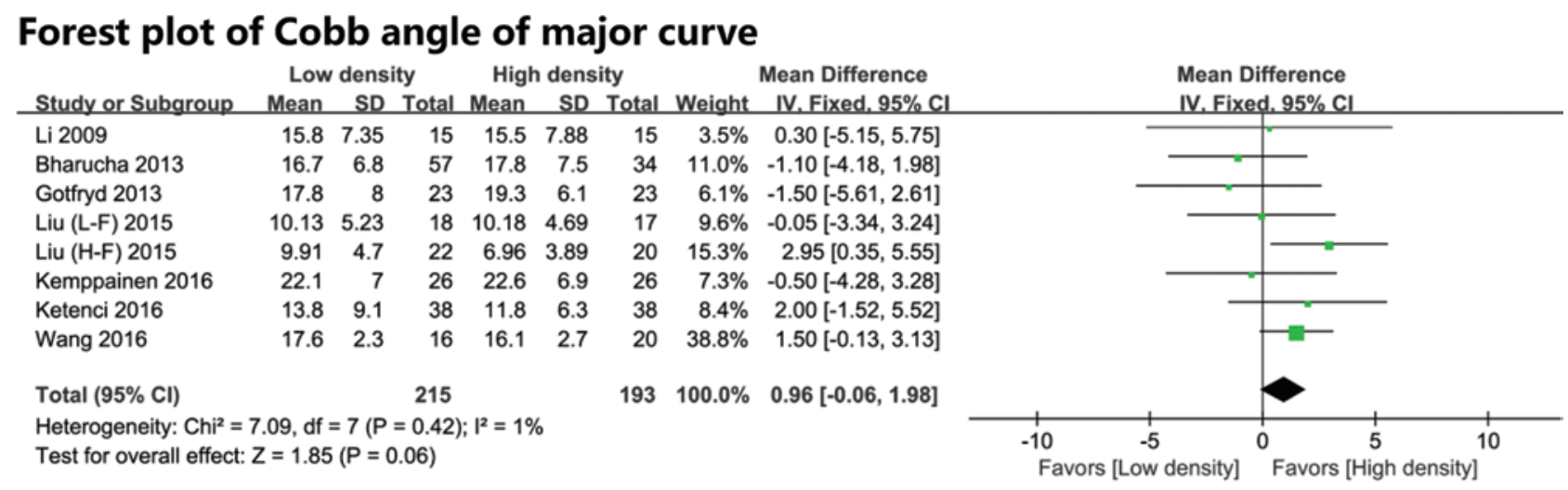

\section{Forest plot of major curve correction}

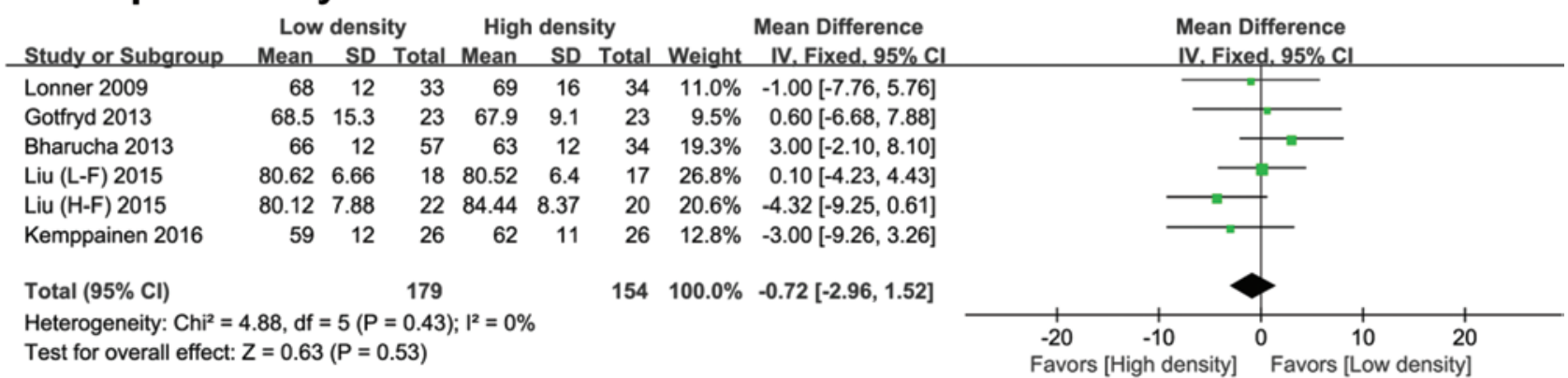

\section{Forest plot of thoracic kyphosis}

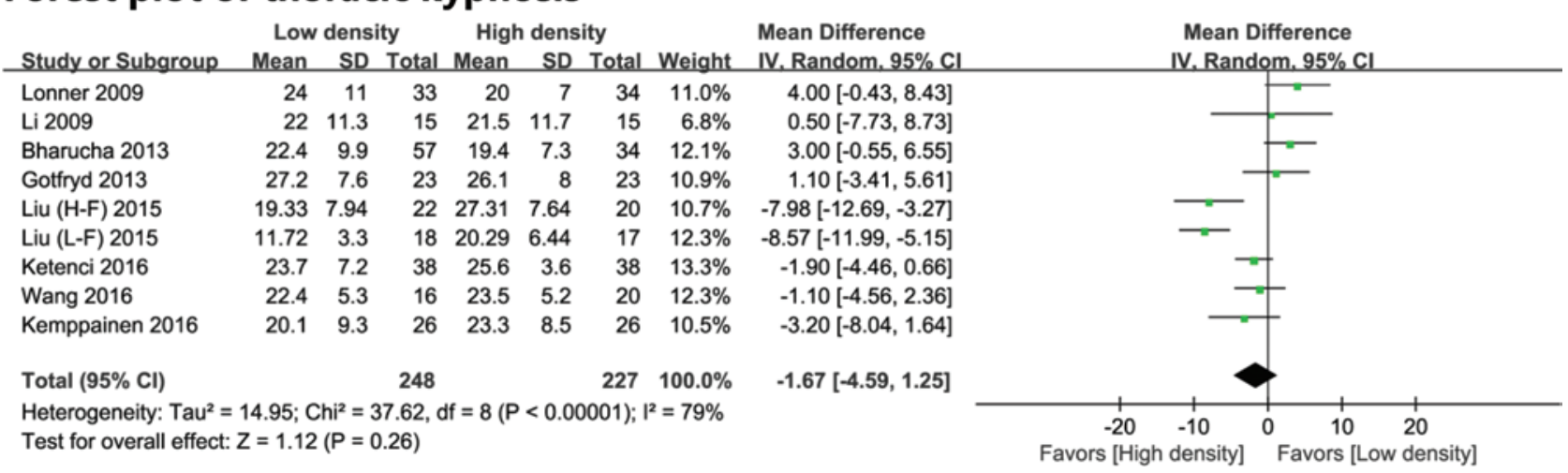

FIG. 3. Forest plots of radiographic outcomes. ${ }^{2,8,11,12,19-21,31}$ Means, standard deviations (SDs), and mean differences are given in degrees. The columns labeled "Total" show the total numbers of participants in the given group and study. $\mathrm{H}-\mathrm{F}=$ high-stiffness group (titanium rod diameter $6.35 \mathrm{~mm}$ ); IV = independent variable; L-F = low-stiffness group (titanium rod diameter $5 \mathrm{~mm}$ ). Figure is available in color online only.

recorded in either group in the studies by Morr et al. ${ }^{23}$ and Ketenci et al. ${ }^{12}$ Instrumentation-related complications were the most common and included malposition, prominent instrumentation, broken pedicle screw, and proximal junctional kyphosis. Wound infection (deep or superficial) was another common complication. Detailed information on complications is shown in Table 3.

\section{Discussion}

Instead of a critical threshold to distinguish LD and HD groups, the primary principle of division in this study was based on the relative screw density (defined as the number of screws per treated level), which was reported in 9 studies. The other 3 studies only described screw patterns, with no specific screw density recorded, and patients were grouped according to the described screw techniques, such as consecutive versus interval/skipped pedicle screw fixation.

Both LD and HD screw constructs achieved excellent coronal correction. No significant differences were found in pooled data on the Cobb angle of major curve $(p=0.06$, $I^{2}=1 \%$ ), and the same result was reported in the studies by Tsirikos and Subramanian, ${ }^{30}$ Tao et al., ${ }^{29}$ and Morr et al. ${ }^{23}$ ( $p=0.86, p=0.19$, and $p=0.35$, respectively). In addition, analysis of pooled data showed comparable major curve correction in the LD and HD groups $\left(\mathrm{p}=0.53, I^{2}=\right.$ $0 \%$ ), and Auerbach et al., ${ }^{1}$ Tsirikos and Subramanian, ${ }^{30}$ and Morr et al. ${ }^{23}$ concluded that there were no differences in major curve correction $(\mathrm{p}=0.56, \mathrm{p}=0.85$, and $\mathrm{p}=0.92$, respectively). These results fell in line with a number of stud- 


\section{Forest plot of operative time}

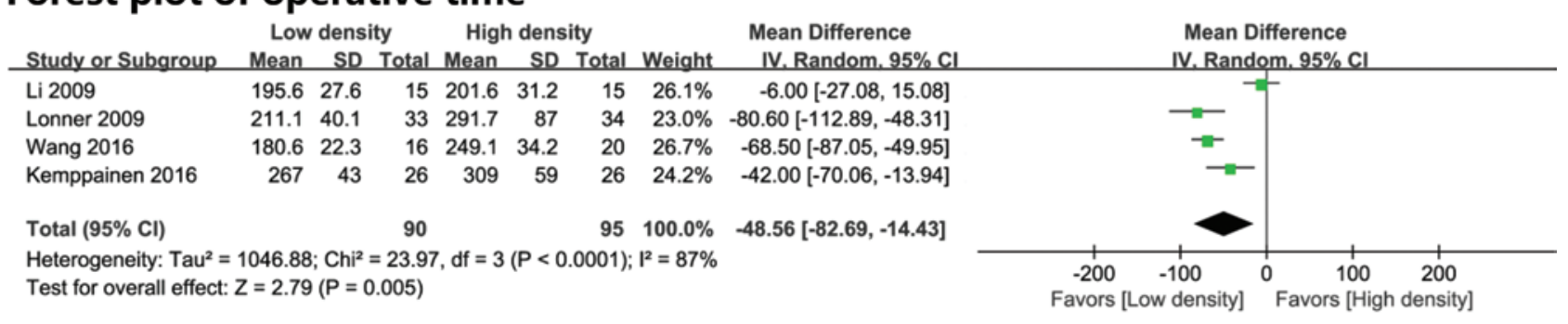

\section{Forest plot of blood loss}

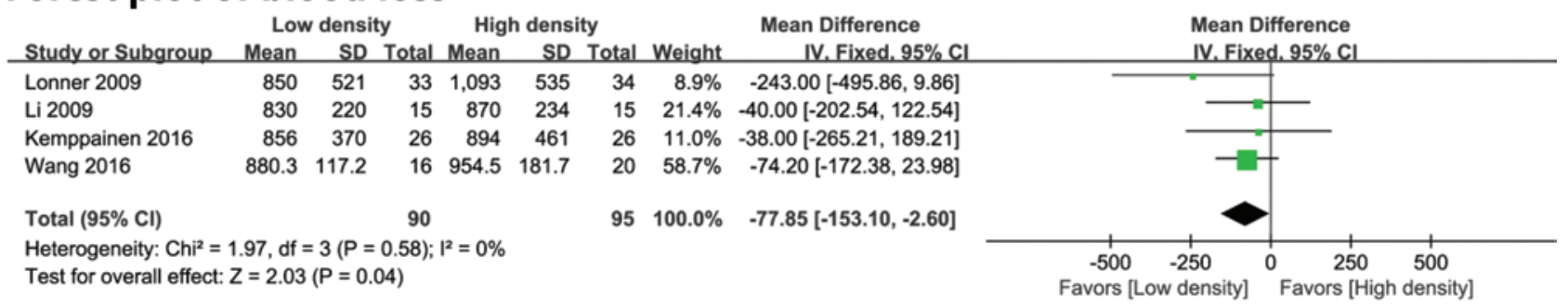

\section{Forest plot of hospital charges}

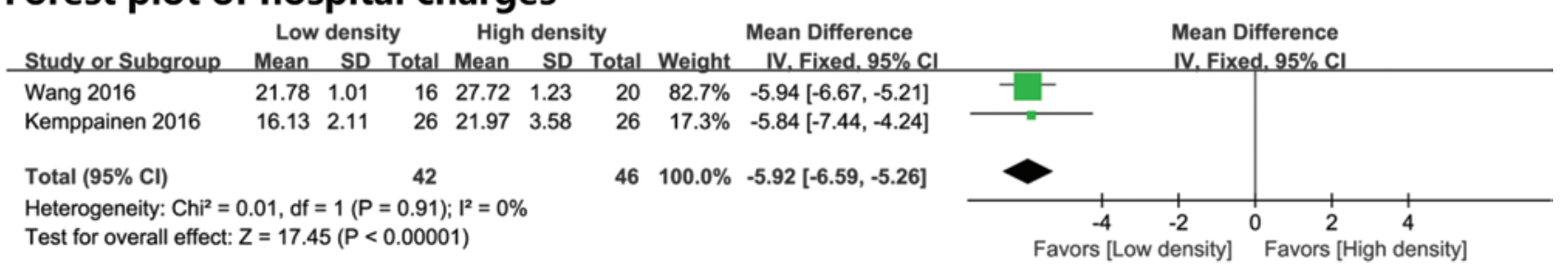

FIG. 4. Forest plots of perioperative outcomes.11,19,21,31 Values for time are in minutes. Values for blood loss are in milliliters. Values for hospital charges are in thousands of dollars. Figure is available in color online only.

ies. ${ }^{3,18,24,33}$ Min et al. ${ }^{22}$ evaluated 48 consecutive AIS patients with a minimum of 10 years' follow-up and concluded that an implant density of 50\% (an average of 1 pedicle screw per vertebra) was sufficient to achieve a long-term stable correction. In addition, biomechanical comparison of alternative screw densities for AIS has indicated that increased numbers of pedicle screws did not provide a higher degree of correction. ${ }^{26,32}$ Furthermore, an international consensus suggested that an implant density less than $80 \%$ was optimal (73\% vs 27\%) for moderate curves with a Cobb angle of $40^{\circ}-70^{\circ} .5$

As to sagittal correction, pooled data on thoracic kyphosis were available in 8 studies, involving a total of 475 patients, and no significant differences were found $(\mathrm{p}=$ $0.26, I^{2}=79 \%$ ). The same conclusion was recorded in 2 additional studies $(\mathrm{p}=0.15$ and $\mathrm{p}=0.37)$. $^{23,30}$ The heterogeneity test of thoracic kyphosis revealed significant heterogeneity $\left(I^{2}=79 \%\right)$. AIS is a 3 -dimensional deformity (involving the coronal, sagittal, and transverse planes). In most controlled studies, the baseline of the major curve was limited to a moderate range of $40^{\circ}-70^{\circ}$. However, with the regulation of the coronal plane, it was difficult to avoid some disparities in baseline thoracic kyphosis in the studies and, consequently, in the outcome with respect to thoracic kyphosis.

LD screw constructs were associated with better perioperative outcomes than HD constructs. Reduced operative time ( $\left.\mathrm{p}=0.005, I^{2}=87 \%\right)$, blood loss $\left(\mathrm{p}=0.04, I^{2}\right.$ $=0 \%)$, and hospital charges $\left(\mathrm{p}<0.00001, I^{2}=0 \%\right)$ were found in the LD group. These were completely consistent with the conclusions of Tsirikos and Subramanian, who reviewed 212 consecutive cases involving patients with AIS and concluded that implant cost was reduced by a mean of $35 \%$ in the LD group due to the reduced number of pedicle screws. ${ }^{30}$ Other authors have expressed concern over implant costs and have reported similar results. ${ }^{25,33}$ Furthermore, Larson et al. ${ }^{15}$ explored the national all-payer hospital inpatient database in the US and analyzed the annual costs for AIS patients. By changing the HD screw pattern to LD, the total cost of AIS surgery in the US would effectively be reduced by $4 \%-7 \%$ ( $\$ 11$ million- $\$ 20$ million). Regrettably, significant heterogeneity existed in operative time $\left(I^{2}=87 \%\right)$. As we know, spinal deformity surgery is one of the most difficult types of surgery, and the number of implanted pedicle screws may range from 10 to 20 , or even more. The operative time largely depends on the surgeon's skill and experience, and the standardization of operative time is difficult.

Six studies, $1,2,8,12,23,30$ involving a total of 419 patients, reported QOL outcomes at the last follow-up. Regrettably, the pooling analysis was restricted due to the different methodologies of evaluating QOL outcomes, such as the SRS-22 questionnaire, the SRS-30 questionnaire, and the SAQ. SRS-22 scores were reported in 5 studies and SRS-30 scores were reported in 1 study. The 6 studies had very consistent results: no significant differences were found between LD and HD screw constructs in SRS-22 scores and SRS-30 scores, including function/ 
TABLE 3. QOL outcomes and complications of the included studies

\begin{tabular}{|c|c|c|c|c|}
\hline Authors \& Year & Group & $\begin{array}{l}\text { No. of } \\
\text { Cases }\end{array}$ & Complications & QOL Outcomes \\
\hline \multirow{2}{*}{$\begin{array}{l}\text { Lonner et al., } \\
2009\end{array}$} & LD & 33 & NR & NR \\
\hline & HD & 34 & NR & NR \\
\hline \multirow{2}{*}{$\begin{array}{l}\text { Auerbach et al., } \\
2009\end{array}$} & LD & 27 & 1 instr-related complications & \multirow[t]{2}{*}{ Similar total SRS-22 scores } \\
\hline & $\mathrm{HD}$ & 27 & 3 instr-related complications & \\
\hline \multirow[t]{2}{*}{ Li et al., 2009} & LD & 15 & 1 pseudarthrosis w/ instr failure; 1 postop SWI & NR \\
\hline & $H D$ & 15 & 2 DWls & NR \\
\hline \multirow[t]{2}{*}{ Tao et al., 2010} & LD & 24 & No instances of crankshaft phenom & \multirow[t]{2}{*}{ NR } \\
\hline & HD & 22 & No instances of crankshaft phenom & \\
\hline \multirow[t]{2}{*}{$\begin{array}{l}\text { Tsirikos \& Subra- } \\
\quad \text { manian, } 2012\end{array}$} & LD & 161 & $\begin{array}{l}1 \text { deep inf; } 1 \text { wound inf; } 1 \text { transient neuro- } \\
\text { praxia; } 1 \text { SMA syndrome }\end{array}$ & \multirow[t]{2}{*}{ Similar total SRS-22 scores (LD 4.5, HD 4.5) } \\
\hline & $\mathrm{HD}$ & 51 & $\begin{array}{l}1 \text { transient loss of op spinal cord monitoring } \\
\text { traces; } 1 \text { deep inf; } 1 \text { prominent instr }\end{array}$ & \\
\hline \multirow{2}{*}{$\begin{array}{l}\text { Gotfryd \& Avanzi, } \\
\quad 2013\end{array}$} & LD & 23 & No complications were observed & \multirow{2}{*}{$\begin{array}{l}\text { Similar SRS-30 scores for function }(p=0.48) \text {, pain }(p= \\
0.09) \text {, cosmesis }(p=0.83) \text {, mental health }(p=0.07), \& \\
\text { overall satisfaction }(p=0.54)\end{array}$} \\
\hline & $\mathrm{HD}$ & 23 & $\begin{array}{l}1 \text { noninfected op wound seroma; } 1 \text { decompen- } \\
\text { sation of trunk to left side }\end{array}$ & \\
\hline \multirow{2}{*}{$\begin{array}{l}\text { Bharucha et al., } \\
2013\end{array}$} & LD & 57 & 3 instr-related revisions & \multirow{2}{*}{$\begin{array}{l}\text { Similar SRS-22 scores for appearance }(p=0.23) \text {, activity }(p \\
\quad=0.84), \text { pain }(p=0.34) \text {, mental }(p=0.74) \text {, satisfaction }(p= \\
0.88), \& \text { total }(p=0.68)\end{array}$} \\
\hline & $\mathrm{HD}$ & 34 & No complication observed & \\
\hline \multirow{2}{*}{$\begin{array}{l}\text { Liu et al., } 2015 \\
\qquad(\mathrm{~L}-\mathrm{F})\end{array}$} & LD & 18 & NR & \multirow{2}{*}{$\begin{array}{l}\text { NR } \\
\text { NR }\end{array}$} \\
\hline & $\mathrm{HD}$ & 17 & NR & \\
\hline \multirow{2}{*}{$\begin{array}{l}\text { Liu et al., } 2015 \\
\qquad(\mathrm{H}-\mathrm{F})\end{array}$} & LD & 22 & NR & \multirow{2}{*}{$\begin{array}{l}N R \\
N R\end{array}$} \\
\hline & $\mathrm{HD}$ & 20 & NR & \\
\hline \multirow[t]{2}{*}{ Morr et al., 2015} & LD & 20 & No screw-related complications & \multirow{2}{*}{$\begin{array}{l}\text { Similar total SRS-22 scores (LD 94.3, HD 96, } p=0.34) \text {, } \\
\text { pain }(p=0.16) \text {, self-image }(p=0.54) \text {, function }(p=0.29) \text {, } \\
\text { satisfaction }(p=0.20)\end{array}$} \\
\hline & $\mathrm{HD}$ & 20 & No screw-related complications & \\
\hline \multirow[t]{2}{*}{ Wang et al., 2016} & LD & 16 & No complication observed & \multirow{2}{*}{$\begin{array}{l}\text { NR } \\
\text { NR }\end{array}$} \\
\hline & $\mathrm{HD}$ & 20 & 1 wound inf & \\
\hline \multirow{2}{*}{$\begin{array}{l}\text { Ketenci et al., } \\
2016\end{array}$} & LD & 38 & No intraop or postop complications observed & \multirow{2}{*}{$\begin{array}{l}\text { Better SAQ scores }(p=0.035) \text { in HD group; similar total } \\
\text { SRS-22 scores }(p=0.102) \& \text { total SAQ scores }(p=0.426)\end{array}$} \\
\hline & $\mathrm{HD}$ & 38 & No intraop or postop complications observed & \\
\hline \multirow[t]{2}{*}{$\begin{array}{l}\text { Kemppainen et } \\
\quad \text { al., } 2016\end{array}$} & LD & 26 & $\begin{array}{l}1 \text { superficial wound dehiscence; } 1 \text { delayed } \\
\text { deep inf; } 1 \text { unilat broken PS }\end{array}$ & NR \\
\hline & $\mathrm{HD}$ & 26 & $\begin{array}{l}1 \text { prox junctional kyphosis; } 1 \text { metallosis at low- } \\
\text { est instrumented vertebra; } 1 \text { deep inf }\end{array}$ & NR \\
\hline
\end{tabular}

$\mathrm{DWI}=$ deep wound infection; inf = infection; instr = instrumentation; op = operative; phenom = phenomenon; prox = proximal; SMA = superior mesenteric artery; SWI = superficial wound infection.

activity, pain, self-image, mental health, satisfaction, and total scores. Although Ketenci et al. ${ }^{12}$ reported that the HD group achieved a significantly better score in the SAQ appearance domain $(\mathrm{p}=0.035)$, the 1-year follow-up period of this study might not be long enough to offer powerful evidence. Moreover, Yang et al. ${ }^{33}$ reviewed 58 Lenke Type 1 AIS cases and concluded that increasing the number of implants did not improve cosmetic outcomes.

Screw-related complications are a primary consideration for orthopedic surgeons. Although a systematic review analyzed 13,536 pedicle screws placed in 1353 pediatric patients and the overall placement accuracy rate was satisfactory $(94.9 \%)$, ${ }^{16}$ if neurological complications or spinal cord injuries occurred, the consequences could be disastrous. Diab et al. ${ }^{6}$ reviewed 1301 consecutive surgical cases of AIS and reported that the overall neurological complication rate was $0.69 \%$. In our pooled statistical analysis of complications, which included 521 patients, no significant differences were observed between the LD and HD groups with respect to neurological complications $\left(\mathrm{p}=0.29, I^{2}=0 \%\right)$, and screw-related complications were the most common. We have reason to believe that fewer pedicle screws may potentially decrease the risk of screw-related complications, and more research exploring the correlation between screw density and screw-related complications is needed.

Some limitations should not be ignored in this study. First, instead of a critical threshold to distinguish the 2 groups, the definition of LD and HD screw constructs was relative, which was restricted by the different methodologies of the included studies. Second, almost all included studies were retrospective, which caused a large reduction in the level of evidence for the pooled statistical analysis. Additional randomized controlled trials should be conducted. Third, the pooled sample size was not large enough to provide strong evidence, and research with larger samples is needed. 


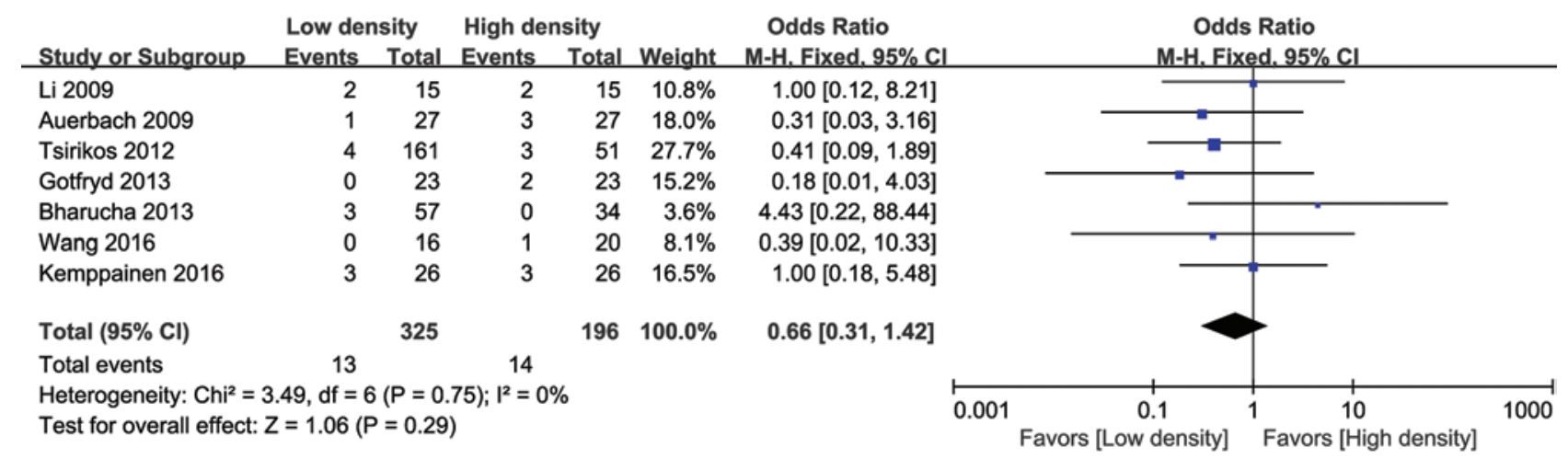

FIG. 5. Forest plot of complications. ${ }^{1,2,8,11,19,30,31} \mathrm{M}-\mathrm{H}=$ Mantel-Haenszel. Figure is available in color online only.

\section{Conclusions}

LD and HD screw constructs both achieve satisfactory radiographic and QOL outcomes with few complications in AIS patients. The increased costs in HD screw constructs associated with the higher number of pedicle screws should be seriously considered, as health economics plays an increasingly deterministic role in the provision of health care.

This study supports the use of LD screw constructs for patients with moderate AIS because it results in reduced operative time, blood loss, and hospital charges, while maintaining radiographic and QOL outcomes and complication rates similar to those for the use of HD screw constructs in these patients.

\section{References}

1. Auerbach J, Lonner B, Kean K: Low-density vs. high-density thoracic pedicle screw constructs in adolescent idiopathic scoliosis: is more better? Spine J 9 (10 Suppl):168S, 2009 (Abstract)

2. Bharucha NJ, Lonner BS, Auerbach JD, Kean KE, Trobisch PD: Low-density versus high-density thoracic pedicle screw constructs in adolescent idiopathic scoliosis: do more screws lead to a better outcome? Spine J 13:375-381, 2013

3. Chen J, Yang C, Ran B, Wang Y, Wang C, Zhu X, et al: Correction of Lenke 5 adolescent idiopathic scoliosis using pedicle screw instrumentation: does implant density influence the correction? Spine (Phila Pa 1976) 38:E946-E951, 2013

4. Crawford AH, Lykissas MG, Gao X, Eismann E, Anadio J: All-pedicle screw versus hybrid instrumentation in adolescent idiopathic scoliosis surgery: a comparative radiographical study with a minimum 2-year follow-up. Spine (Phila Pa 1976) 38:1199-1208, 2013

5. de Kleuver M, Lewis SJ, Germscheid NM, Kamper SJ, Alanay A, Berven SH, et al: Optimal surgical care for adolescent idiopathic scoliosis: an international consensus. Eur Spine J 23:2603-2618, 2014

6. Diab M, Smith AR, Kuklo TR: Neural complications in the surgical treatment of adolescent idiopathic scoliosis. Spine (Phila Pa 1976) 32:2759-2763, 2007

7. Gebhart S, Alton TB, Bompadre V, Krengel WF: Do anchor density or pedicle screw density correlate with short-term outcome measures in adolescent idiopathic scoliosis surgery? Spine (Phila Pa 1976) 39:E104-E110, 2014

8. Gotfryd AO, Avanzi O: Randomized clinical study on surgical techniques with different pedicle screw densities in the treatment of adolescent idiopathic scoliosis types Lenke 1A and 1B. Spine Deform 1:272-279, 2013
9. Hwang SW, Samdani AF, Marks M, Bastrom T, Garg H, Lonner B, et al: Five-year clinical and radiographic outcomes using pedicle screw only constructs in the treatment of adolescent idiopathic scoliosis. Eur Spine J 22:1292-1299, 2013

10. Kamerlink JR, Quirno M, Auerbach JD, Milby AH, Windsor L, Dean L, et al: Hospital cost analysis of adolescent idiopathic scoliosis correction surgery in 125 consecutive cases. J Bone Joint Surg Am 92:1097-1104, 2010

11. Kemppainen JW, Morscher MA, Gothard MD, Adamczyk MJ, Ritzman TF: Evaluation of limited screw density pedicle screw constructs in posterior fusions for adolescent idiopathic scoliosis. Spine Deform 4:33-39, 2016

12. Ketenci IE, Yanik HS, Demiroz S, Ulusoy A, Erdem S: Three-dimensional correction in patients with Lenke 1 adolescent idiopathic scoliosis: comparison of consecutive versus interval pedicle screw instrumentation. Spine (Phila Pa 1976) 41:134-138, 2016

13. Kim YJ, Lenke LG, Kim J, Bridwell KH, Cho SK, Cheh G, et al: Comparative analysis of pedicle screw versus hybrid instrumentation in posterior spinal fusion of adolescent idiopathic scoliosis. Spine (Phila Pa 1976) 31:291-298, 2006

14. Kuklo TR, Potter BK, Lenke LG, Polly DW Jr, Sides B, Bridwell KH: Surgical revision rates of hooks versus hybrid versus screws versus combined anteroposterior spinal fusion for adolescent idiopathic scoliosis. Spine (Phila Pa 1976) 32:2258-2264, 2007

15. Larson AN, Polly DW Jr, Ackerman SJ, Ledonio CG, Lonner BS, Shah SA, et al: What would be the annual cost savings if fewer screws were used in adolescent idiopathic scoliosis treatment in the US? J Neurosurg Spine 24:116-123, 2016

16. Ledonio CG, Polly DW Jr, Vitale MG, Wang Q, Richards BS: Pediatric pedicle screws: comparative effectiveness and safety: a systematic literature review from the Scoliosis Research Society and the Pediatric Orthopaedic Society of North America task force. J Bone Joint Surg Am 93:1227-1234, 2011

17. Legarreta CA, Barrios C, Rositto GE, Reviriego JM, Maruenda JI, Escalada MN, et al: Cervical and thoracic sagittal misalignment after surgery for adolescent idiopathic scoliosis: a comparative study of all pedicle screws versus hybrid instrumentation. Spine (Phila Pa 1976) 39:1330-1337, 2014

18. Li J, Cheung KM, Samartzis D, Ganal-Antonio AK, Zhu X, $\mathrm{Li} \mathrm{M}$, et al: Key-vertebral screws strategy for main thoracic curve correction in patients with adolescent idiopathic scoliosis. Clin Spine Surg 29:E434-E441, 2016

19. Li M, Shen Y, Fang X, Ni J, Gu S, Zhu X, et al: Coronal and sagittal plane correction in patients with Lenke 1 adolescent idiopathic scoliosis: a comparison of consecutive versus interval pedicle screw placement. J Spinal Disord Tech 22:251-256, 2009

20. Liu H, Li Z, Li S, Zhang K, Yang H, Wang J, et al: Main 
thoracic curve adolescent idiopathic scoliosis: association of higher rod stiffness and concave-side pedicle screw density with improvement in sagittal thoracic kyphosis restoration. J Neurosurg Spine 22:259-266, 2015

21. Lonner BS, Auerbach JD, Boachie-Adjei O, Shah SA, Hosogane N, Newton PO: Treatment of thoracic scoliosis: are monoaxial thoracic pedicle screws the best form of fixation for correction? Spine (Phila Pa 1976) 34:845-851, 2009

22. Min K, Sdzuy C, Farshad M: Posterior correction of thoracic adolescent idiopathic scoliosis with pedicle screw instrumentation: results of 48 patients with minimal 10-year follow-up. Eur Spine J 22:345-354, 2013

23. Morr S, Carrer A, Alvarez-García de Quesada LI, Rodriguez-Olaverri JC: Skipped versus consecutive pedicle screw constructs for correction of Lenke 1 curves. Eur Spine J 24:1473-1480, 2015

24. Quan GM, Gibson MJ: Correction of main thoracic adolescent idiopathic scoliosis using pedicle screw instrumentation: does higher implant density improve correction? Spine (Phila Pa 1976) 35:562-567, 2010

25. Rushton PR, Elmalky M, Tikoo A, Basu S, Cole AA, Grevitt MP: The effect of metal density in thoracic adolescent idiopathic scoliosis. Eur Spine J 25:3324-3330, 2016

26. Salmingo RA, Tadano S, Fujisaki K, Abe Y, Ito M: Relationship of forces acting on implant rods and degree of scoliosis correction. Clin Biomech (Bristol, Avon) 28:122-128, 2013

27. Samdani AF, Belin EJ, Bennett JT, Pahys JM, Marks MC, Miyanji F, et al: Unplanned return to the operating room in patients with adolescent idiopathic scoliosis: are we doing better with pedicle screws? Spine (Phila Pa 1976) 38:18421847,2013

28. Sudo H, Abe Y, Kokabu T, Ito M, Abumi K, Ito YM, et al: Correlation analysis between change in thoracic kyphosis and multilevel facetectomy and screw density in main thoracic adolescent idiopathic scoliosis surgery. Spine J 16:10491054,2016

29. Tao F, Zhao Y, Wu Y, Xie Y, Li M, Lu Y, et al: The effect of differing spinal fusion instrumentation on the occurrence of postoperative crankshaft phenomenon in adolescent idiopathic scoliosis. J Spinal Disord Tech 23:e75-e80, 2010
30. Tsirikos AI, Subramanian AS: Posterior spinal arthrodesis for adolescent idiopathic scoliosis using pedicle screw instrumentation: does a bilateral or unilateral screw technique affect surgical outcome? J Bone Joint Surg Br 94:1670-1677, 2012

31. Wang F, Xu XM, Lu Y, Wei XZ, Zhu XD, Li M: Comparative analysis of interval, skipped, and key-vertebral pedicle screw strategies for correction in patients with Lenke Type 1 adolescent idiopathic scoliosis. Medicine (Baltimore) 95:e3021, 2016

32. Wang X, Aubin CE, Robitaille I, Labelle H: Biomechanical comparison of alternative densities of pedicle screws for the treatment of adolescent idiopathic scoliosis. Eur Spine J 21:1082-1090, 2012

33. Yang S, Jones-Quaidoo SM, Eager M, Griffin JW, Reddi V, Novicoff W, et al: Right adolescent idiopathic thoracic curve (Lenke $1 \mathrm{~A}$ and $\mathrm{B}$ ): does cost of instrumentation and implant density improve radiographic and cosmetic parameters? Eur Spine J 20:1039-1047, 2011

\section{Disclosures}

The authors report no conflict of interest concerning the materials or methods used in this study or the findings specified in this paper.

\section{Author Contributions}

Conception and design: Xia, M Luo, Wang. Acquisition of data: M Luo, Wang, Shen. Analysis and interpretation of data: M Luo, Wang, Shen. Drafting the article: M Luo, Wang. Critically revising the article: Xia, Wang. Reviewed submitted version of manuscript: Xia, M Luo, Wang, X Luo. Approved the final version of the manuscript on behalf of all authors: Xia. Statistical analysis: M Luo. Administrative/technical/material support: Xia, X Luo.

\section{Correspondence}

Lei Xia, Institute of Spinal Deformity, The First Affiliated Hospital of Zhengzhou University, No. 1 Jianshe Dong Rd., Zhengzhou, Henan 450052, P. R. China. email: xialeigu1@126.com. 